\title{
Protein conformational flexibility prediction using machine learning
}

\author{
Oleg Trott ${ }^{1}$, Keri Siggers ${ }^{2}$, Burkhard Rost, Arthur G. Palmer III* \\ Department of Biochemistry and Molecular Biophysics, The Columbia University College of Physicians and Surgeons, \\ Columbia University, Box 36, 630 West 168th Street, New York, NY 10032, USA \\ Received 24 July 2006; revised 15 December 2007 \\ Available online 1 February 2008
}

\begin{abstract}
Using a data set of 16 proteins, a neural network has been trained to predict backbone ${ }^{15} \mathrm{~N}$ generalized order parameters from the three-dimensional structures of proteins. The final network parameterization contains six input features. The average prediction accuracy, as measured by the Pearson's correlation coefficient between experimental and predicted values of the square of the generalized order parameter is $>0.70$. Predicted order parameters for non-terminal amino acid residues depends most strongly on the local packing density and the probability that the residue is located in regular secondary structure.
\end{abstract}

(C) 2008 Elsevier Inc. All rights reserved.

Keywords: Fibronectin; FREAC-11; Generalized order parameter; NMR; Neural network; Relaxation; Tenascin

\section{Introduction}

Dynamical processes in proteins are believed to be closely related to protein function, including ligand-binding, catalysis, and folding, even though this relationship is not yet understood in great detail [1]. Moreover, information about protein conformational flexibility is becoming important in drug design [2]. Thus, considerable importance exists in the related problems of elucidating the microscopic factors that determine protein conformational flexibility and of predicting flexibility from sequence or structural data.

Theoretical assessments of protein flexibility can derive from computational simulations with atomistic and mechanistic detail [3] or from more abstract approaches [4,5]. Theoretical approaches can be free-standing or aimed at

\footnotetext{
* Corresponding author. Fax: +1 2123056949.

E-mail address: agp6@columbia.edu (A.G. Palmer III).

${ }^{1}$ Present address: Department of Molecular Biology, The Scripps Research Institute, 10550 N. Torrey Pines Road, La Jolla, CA 92037, USA.

${ }^{2}$ Present address: Department of Medicine, Division of Infectious Diseases, Massachusetts General Hospital, Harvard Medical School, Cambridge, MA 02139, USA.
}

interpretation of experimental measurements of protein flexibility, such as crystallographic B-factors $[6,7]$.

NMR spin relaxation experiments are widely applied for the study of the dynamics of macromolecules $[8,9]$. NMR spin relaxation data has been collected for various proteins by a number of different research groups, and some of these data have been compiled into publicly accessible data banks $[10,11]$. Most commonly, laboratory frame relaxation experiments conducted for ${ }^{15} \mathrm{~N}[12,13]$ or ${ }^{2} \mathrm{H}[14,15]$ spins have been used to determine the square of the generalized order parameter, $S^{2}$, [16] for backbone amide or side chain methyl groups, respectively [8].

A number of authors have used the availability of such NMR data as the basis for further studies of conformational flexibility of proteins (for a recent review, see [17]). Order parameters derived from NMR have been compared with other experimental and theoretical measures of protein flexibility, including crystallographic B-factors [18], order parameters obtained from fluorescence anisotropy decay measurements $[19,20]$, and order parameters obtained from molecular dynamics (MD) simulations $[21,22]$. Correlations have been uncovered between order parameters and molecular features, such as secondary structural elements and amino acid side chain volumes 
$[10,23]$ and amino acid sequence conservation [24]. Abergel and Bodenhausen used a model comprising a network of coupled rotators to predict generalized order parameters from protein structures [25]. Using a database of backbone amide order parameters, Zhang and Brüschweiler empirically devised a simple analytic method for predicting generalized order parameters from static three-dimensional protein structures [26] and subsequently extended their approach to generalized order parameters for side-chain methyl side groups [27] and to a contact-weighted elastic network model [28]. Schlessinger and Rost used a neural network to predict B-factors and generalized order parameters from protein sequences [7].

Our goal in the present work is to devise a systematic knowledge-based method for predicting picosecond to nanosecond protein backbone flexibility as described by generalized order parameters obtained from NMR measurements. We are interested in "learning" $S^{2}$ as a function of structure from "examples" without necessarily looking in detail into the physics of the process. As "examples", we use backbone ${ }^{15} \mathrm{~N}$ order parameters deposited into the Indiana Dynamics Database (IDD) [10] and the BioMagResBank (BMRB) [11] and the corresponding 3D structures from the Protein Data Bank (PDB) [29]. We use a particular type of neural network typically referred to as a multi-layered feed-forward network (or as a multi-layer perceptron) with one hidden layer [30] to optimize the prediction of $S^{2}$ given the set of "examples". We anticipate that similar approaches also can be applied to predicting slower time scale dynamic properties accessible to NMR experiments [9].

\section{Methods}

\subsection{Order parameter}

The angular distribution of the orientations of the backbone $\mathrm{N}-\mathrm{H}$ bond vector on the picosecond to nanosecond time scale is described using the square of the generalized order parameter from the Lipari-Szabo model-free formalism [16]

$S^{2}=(4 \pi / 5) \sum_{m=-2}^{2}\left|\left\langle Y_{2, m}(\Omega)\right\rangle\right|^{2}$,

where $Y_{2, m}(\Omega)$ are the second order spherical harmonics, and $\Omega$ describes the orientation of the $\mathrm{N}-\mathrm{H}$ bond vector in the protein-attached coordinate system. In the limiting case of completely isotropic orientation of the bond vector with respect to the body of the molecule, $S^{2}=0$. Alternatively, $S^{2}=1$, if the orientation is fixed.

\subsection{Data banks}

Table 1 lists the PDB, IDD and BMRB entries that were used in the present work. The data set contains two pairs of closely related proteins: 1clb and 1cdn are the apo and $\left(\mathrm{Cd}^{2+}\right)_{1}$ forms of calbindin $\mathrm{D}_{9 k}$, and 1xoa and 1xob are oxidized and reduced forms of thioredoxin. As discussed by Goodman et al. [10], some of the systematic differences in $S^{2}$ between different data sets are due to the differences in the ways the data were collected and analyzed. Normalization of $S^{2}$ reduces this artificial variation. Goodman et al. divide each $S^{2}$ by the average value of $S^{2}$ for the protein in which the $\mathrm{N}-\mathrm{H}$ group resides. We employ a similar linear transformation. However, under the assumption that the true average order parameter of residues in the secondary structure is likely to vary less among different proteins than the order parameter averaged over all residues of the protein, we use the former as the reference point, "normalizing" each database entry so that the average value of $S^{2}$ for residues in the secondary structure becomes 0.86 . This value is close to the canonical value measured in proteins for $\mathrm{N}-\mathrm{H}$ moieties in secondary structure elements when

Table 1

Dataset composition

\begin{tabular}{|c|c|c|c|c|c|c|}
\hline Index & PDB entry & Chain ID & Residues & Database entry & $S^{2}$ values & Relatives \\
\hline 1 & $3 \mathrm{CI} 2$ & & 66 & IDD 1 & 58 & \\
\hline 2 & 1 CLB & & 76 & IDD 2 & 72 & $1 \mathrm{CDN}$ \\
\hline 3 & $1 \mathrm{CDN}$ & & 76 & IDD 3 & 71 & $1 \mathrm{CLB}$ \\
\hline 4 & $1 \mathrm{STG}$ & & 149 & IDD 4 & 106 & \\
\hline 5 & $2 \mathrm{BBN}$ & $\mathrm{A}$ & 148 & IDD 5 & 114 & \\
\hline 6 & $1 \mathrm{XOB}$ & & 108 & IDD 7 & 95 & $1 \mathrm{XOA}$ \\
\hline 7 & $1 \mathrm{XOA}$ & & 108 & IDD 8 & 96 & $1 \mathrm{XOB}$ \\
\hline 8 & $1 \mathrm{GPR}$ & & 162 & IDD 9 & 128 & \\
\hline 9 & $1 \mathrm{BVE}$ & A & 99 & IDD 10 & 78 & \\
\hline 10 & 1 ITM & & 130 & IDD 13 & 113 & \\
\hline 11 & $1 \mathrm{KUN}$ & & 58 & IDD 25 & 51 & \\
\hline 12 & $2 \mathrm{FSP}$ & & 124 & IDD 31 & 109 & \\
\hline 13 & $1 \mathrm{NGL}$ & A & 179 & BMR 4267 & 147 & \\
\hline 14 & $1 \mathrm{VRF}$ & $\mathrm{A}$ & 147 & BMR 4096 & 138 & \\
\hline 15 & 1D2B & A & 126 & BMR 5154 & 102 & \\
\hline 16 & $1 \mathrm{D} 3 \mathrm{Z}$ & A & 76 & IDD 11 & 70 & \\
\hline
\end{tabular}


an effective bond length of $1.02 \AA$ is used in the data analysis.

\subsection{Supervised learning}

"Learning from examples" constitutes what is known as the supervised learning or the function approximation problem [30], which is informally stated as follows: given an unknown function $f(\vec{x})$ and a training set $\left\{\left(\vec{x}_{i}, \vec{y}_{i}\right)\right\}_{i=1}^{\mathrm{N}}$, for which $\vec{y}_{i} \approx f\left(\vec{x}_{i}\right)$, find an approximation of the unknown function $f(\vec{x})$. This approximation is typically obtained from an adequately general parametrization $F(\vec{x}, \vec{w})$ by optimizing parameters $\vec{w}$. Supervised learning problems can be solved using artificial neural networks. We use a special kind of a neural network called a multilayered feed-forward network with one hidden layer [30]. This network architecture corresponds to the parametrization expression:

$F=s_{2}\left(\mathrm{~W}_{2} s_{1}\left(\mathrm{~W}_{1} \vec{x}+\vec{b}_{1}\right)+\vec{b}_{2}\right)$,

where weight matrices $\mathrm{W}_{1}, \mathrm{~W}_{2}$ and bias vectors $\vec{b}_{1}, \vec{b}_{2}$ are the parameters adjusted to fit the training data, and $s_{1}(x)$, $s_{2}(x)$ are the transfer functions, which we choose to be an elementwise application of the sigmoid function:

$s_{1}(x)=s_{2}(x)=\operatorname{sigm}(x)=1 /\left(\mathrm{e}^{-x}+1\right)$.

The function being approximated is the value of $S^{2}$ for the $i$ th amino acid residue; therefore, $f(\vec{x})$ is a scalar. The universal approximation theorem [30] implies that this parametrization can approximate any continuous function with values within $[0,1]$ to any given accuracy, if sufficiently large dimensionalities of $\mathrm{W}_{1}, \mathrm{~W}_{2}, b_{1}$ and $b_{2}$ are allowed.

\subsection{Features}

Instead of using the 3D structure of the protein in some machine-readable form as inputs in the training set, we extracted features of the 3D structure that appear to be statistically related to conformational flexibility and use those features as inputs. Statistical correlations between features and $S^{2}$ were measured using the Pearson's correlation coefficient, defined by

$C_{x y}=\frac{\overline{x y}-\bar{x} \bar{y}}{\sigma_{x} \sigma_{y}}$

for two variables $x$ (=experimental $\left.S^{2}\right)$ and $y$ (=feature or predicted $S^{2}$ ) in which the bars indicate averaging and $\sigma_{x}$ and $\sigma_{y}$ are the standard deviations in the two variables. These statistics were calculated over the number of generalized order parameters available for each protein. Correlations were considered between $S^{2}$ for the $\mathrm{N}-\mathrm{H}$ bond vector of the $i$ th residue and features for the $i$ th residue and flanking residues in the amino acid sequence. "Position" denotes the position of the residue in the protein chain for which the feature is calculated relative to the position of the residue for which $S^{2}$ is measured. Thus, a correlation coefficient reported for feature $p$ and position $k$ indicates that the correlation coefficient was calculated using Eq. (4) with $x=S_{i}^{2}$ and $y=p_{i+k}$ for $i=1, N$ and $N$ is the number of amino acid residues for which data are available. Ranges of $k$ from -6 to +6 were examined. Correlations between features and $S^{2}$ were calculated both by pooling all data for all proteins and by averaging the correlations obtained for individual proteins. The features examined, which were chosen based on intuition and the work of others $[6,26]$, are described below.

The DSSP (dictionary of secondary structure of proteins) program [31] classifies the secondary structure state of each residue in the protein as helix $\left(3_{10}, \mathrm{G} ; \alpha, \mathrm{H} ; \pi, \mathrm{I}\right)$, extended sheet $(\mathrm{E}), \beta$ bridge $(\mathrm{B})$, turn $(\mathrm{T})$, bend $(\mathrm{S})$, or "other" (L). The continuum secondary structure assignment DSSPcont [23] extends this method by capturing "uncertainties" of DSSP assignments and assigning an eight-dimensional vector to each residue. The vector can be thought of as the probabilities of the respective DSSP assignments. The sum of all eight elements, therefore, equals 1. Each of the eight DSSPcont probabilities was treated as a feature. In addition, the feature "secondary" was defined as the sum of all DSSPcont values except ' $L$ ' and ' $\mathrm{S}$ '.

The feature "BB H-bonds energy" is the energy of the backbone-to-backbone H-bonds that involve a given peptide bond, where the energy is calculated in the same manner as by DSSP.

" $D_{\text {com }}$ " is the distance between the $C_{\alpha}$ atom and the center of mass of the non-hydrogen atoms of the protein.

"Residue size" is the number of all non-hydrogen atoms in the residue.

"Tail $M$ " equals 1 if the peptide bond, to which the $\mathrm{N}-\mathrm{H}$ bond belongs, is $M$ or fewer residues away from $\mathrm{N}$ - or Cterminus, and 0 otherwise. For example, in a protein chain with residues numbered from 1 to 100 , for residues 3 and 99, "Tail $M=1$ " will be 0 , while "Tail $M=2$ " will be 1 .

"Loop left" and "Loop right" show the extent of the non-secondary (loop) structure towards the $\mathrm{N}$ - and C-terminus, respectively. For a given residue, "Loop right" is one-tenth times the relative position towards the C-terminus of the first residue with a "secondary" feature (defined above) greater than 0.95 (or 95\%). If the residue currently of interest is in regular secondary structure, "Loop right" is 0.0 . If such residue is not found (due to a chain break) or is found more than 10 residues away, "Loop right" is 1.0. The definition of "Loop left" is analogous.

"Bend $(-m, 0, m)$ " is the cosine of the angle formed by vectors $\mathrm{C}_{\alpha}(i+k-m) \mathrm{C}_{\alpha}(i+k)$ and $\mathrm{C}_{\alpha}(i+k+m) \mathrm{C}_{\alpha}(i+k)$, where $\mathrm{C}_{\alpha}(n)$, is the $\mathrm{C}_{\alpha}$ atoms of the $n$th residue.

Distance-dependent features, " $g\left(r_{k}^{X}\right)$ ", are given by $\sum_{j} f\left(r_{X_{i+k}, j}\right)$, where $r_{X_{i+k}, j}$ is the distance between the $j$ th atom and the atom $X$ in the $(i+k)$ th residue. The summation extends over all heavy atoms, including hetero-atoms, but not water molecules. When $\mathrm{X}=\mathrm{H}$, the reference atom is the amide hydrogen of the $(i+k)$ th residue and heavy atoms in the $(i+k)$ th and $(i+k-1)$ th residues are not 
Table 2

Dataset-wide correlations $100 C_{x y}$ between features and $S^{2}$

\begin{tabular}{|c|c|c|c|c|c|c|c|c|c|c|c|c|c|}
\hline \multirow[t]{2}{*}{ Feature description } & \multicolumn{13}{|c|}{ Position, $k$} \\
\hline & -6 & -5 & -4 & -3 & -2 & -1 & 0 & +1 & +2 & +3 & +4 & +5 & +6 \\
\hline$\overline{\exp \left(-r_{k}^{\mathrm{O}} / 1 \AA\right)}$ & 4.9 & 12.8 & 21.8 & 28.0 & 33.7 & 37.1 & 32.1 & 23.9 & 13.7 & 4.7 & -1.7 & -8.5 & -9.6 \\
\hline $\exp \left(-r_{k}^{\mathrm{O}} / 2 \AA\right)$ & 8.4 & 16.9 & 27.2 & 34.2 & 40.8 & 44.4 & 39.1 & 28.1 & 17.0 & 6.8 & -1.4 & -8.4 & -9.3 \\
\hline $\exp \left(-r_{k}^{\mathrm{O}} / 3 \AA\right)$ & 9.9 & 18.5 & 28.6 & 35.6 & 42.0 & $\underline{45.5}$ & 40.6 & 29.6 & 18.5 & 8.2 & -0.5 & -7.4 & -8.4 \\
\hline $\exp \left(-r_{k}^{\mathrm{O}} / 4 \AA\right)$ & 10.6 & 19.0 & 28.8 & 35.7 & 41.7 & $\overline{45.1}$ & 40.6 & 30.1 & 19.2 & 9.2 & 0.5 & -6.3 & -7.5 \\
\hline $\exp \left(-r_{k}^{\mathrm{O}} / 5 \AA\right)$ & 11.0 & 19.2 & 28.5 & 35.2 & 41.1 & 44.3 & 40.1 & 30.1 & 19.6 & 9.9 & 1.3 & -5.4 & -6.7 \\
\hline $\exp \left(-r_{k}^{\mathrm{O}} / 7 \AA\right)$ & 11.4 & 18.9 & 27.4 & 33.5 & 38.9 & 41.9 & 38.3 & 29.1 & 19.4 & 10.4 & 2.3 & -3.9 & -5.5 \\
\hline $\exp \left(-r_{k}^{\mathrm{O}} / 10 \AA \hat{A}\right)$ & 11.3 & 17.7 & 24.8 & 30.1 & 34.7 & 37.3 & 34.2 & 26.3 & 17.8 & 9.8 & 2.7 & -2.7 & -4.4 \\
\hline $1 / \exp \left(-r_{k}^{\mathrm{O}} / 1 \AA\right)$ & 4.9 & 12.8 & 21.8 & 28.0 & 33.7 & 37.1 & 32.1 & 23.9 & 13.7 & 4.7 & -1.7 & -8.5 & -9.6 \\
\hline $1 / \exp \left(-r_{k}^{\mathrm{O}} / 2 \AA\right)$ & -8.1 & -16.6 & -25.9 & -36.5 & -47.7 & -54.7 & -47.7 & -33.6 & -21.2 & -10.6 & -0.1 & 6.7 & 8.1 \\
\hline $1 / \exp \left(-r_{k}^{\mathrm{O}} / 3 \AA\right)$ & -12.1 & -20.9 & -31.1 & -41.9 & -52.4 & -58.1 & -52.3 & -38.8 & -25.5 & -13.1 & -1.8 & 5.5 & 7.9 \\
\hline $1 / \exp \left(-r_{k}^{\mathrm{O}} / 4 \AA\right)$ & -14.2 & -23.1 & -33.6 & -44.0 & -53.7 & -58.7 & -53.9 & -41.0 & -27.3 & -14.1 & -2.9 & 4.8 & 7.5 \\
\hline $1 / \exp \left(-r_{k}^{\mathrm{O}} / 5 \AA\right)$ & -15.2 & -24.2 & -34.5 & -44.6 & -53.4 & -58.0 & -53.8 & -41.2 & -27.6 & -14.3 & -3.2 & 4.4 & 7.3 \\
\hline $1 / \exp \left(-r_{k}^{\mathrm{O}} / 7 \AA\right)$ & -15.5 & -24.3 & -34.1 & -43.3 & -51.0 & -54.8 & -50.7 & -38.6 & -25.6 & -13.0 & -2.8 & 4.3 & 6.9 \\
\hline $1 / \exp \left(-r_{k}^{\mathrm{O}} / 10 \AA\right)$ & -13.9 & -21.5 & -30.0 & -37.6 & -43.8 & -46.7 & -42.7 & -31.6 & -20.4 & -9.9 & -1.6 & 4.2 & 6.2 \\
\hline $\exp \left(-r_{k}^{\mathrm{H}} / 1 \AA\right)$ & -4.6 & -1.2 & 3.2 & 9.8 & 16.3 & 26.0 & 33.6 & 33.3 & 31.1 & 22.3 & 16.5 & 10.3 & 8.2 \\
\hline $\exp \left(-r_{k}^{\mathrm{H}} / 2 \AA\right)$ & -1.5 & 3.9 & 11.3 & 20.1 & 28.4 & 39.8 & $\underline{46.5}$ & 43.0 & 36.9 & 27.0 & 17.9 & 8.8 & 5.0 \\
\hline $\exp \left(-r_{k}^{\mathrm{H}} / 3 \AA\right)$ & 0.4 & 6.5 & 14.3 & 23.3 & 31.6 & 41.9 & $\overline{47.4}$ & 43.1 & 35.7 & 26.6 & 17.0 & 7.8 & 3.5 \\
\hline $\exp \left(-r_{k}^{\mathrm{H}} / 4 \AA\right)$ & 1.5 & 7.7 & 15.3 & 24.1 & 32.1 & 41.6 & 46.4 & 42.0 & 34.5 & 25.9 & 16.6 & 7.6 & 3.2 \\
\hline $\exp \left(-r_{k}^{\mathrm{H}} / 5 \AA\right)$ & 2.3 & 8.4 & 15.7 & 24.2 & 31.9 & 40.7 & 45.1 & 40.8 & 33.4 & 25.2 & 16.2 & 7.7 & 3.3 \\
\hline $\exp \left(-r_{k}^{\mathrm{H}} / 7 \AA\right)$ & 3.6 & 9.2 & 15.8 & 23.4 & 30.5 & 38.3 & 42.0 & 38.0 & 31.1 & 23.7 & 15.4 & 7.7 & 3.4 \\
\hline $\exp \left(-r_{k}^{\mathrm{H}} / 10 \AA\right)$ & 4.8 & 9.5 & 15.1 & 21.4 & 27.3 & 33.9 & 36.8 & 33.2 & 27.1 & 20.7 & 13.6 & 7.1 & 3.2 \\
\hline $1 / \exp \left(-r_{k}^{\mathrm{H}} / 1 \AA\right)$ & 2.1 & -2.0 & -7.4 & -15.5 & -22.7 & -33.6 & -38.5 & -40.2 & -34.1 & -25.6 & -16.3 & -11.2 & -8.5 \\
\hline $1 / \exp \left(-r_{k}^{\mathrm{H}} / 2 \AA\right)$ & 0.1 & -6.2 & -14.1 & -24.5 & -34.9 & -48.2 & -53.8 & -51.6 & -43.8 & -32.1 & -20.8 & -11.5 & -6.7 \\
\hline $1 / \exp \left(-r_{k}^{\mathrm{H}} / 3 \AA\right)$ & -1.7 & -9.0 & -17.9 & -29.1 & -39.9 & -53.1 & -58.7 & -55.3 & -46.6 & -34.2 & -22.2 & -11.3 & -5.4 \\
\hline $1 / \exp \left(-r_{k}^{\mathrm{H}} / 4 \AA\right)$ & -2.8 & -10.7 & -19.8 & -30.9 & -41.5 & -54.0 & -59.4 & -55.9 & -46.9 & -34.5 & -22.3 & -11.1 & -4.9 \\
\hline $1 / \exp \left(-r_{k}^{\mathrm{H}} / 5 \AA\right)$ & -3.5 & -11.6 & -20.7 & -31.5 & -41.7 & -53.6 & -58.7 & -55.1 & -45.9 & -33.7 & -21.6 & -10.6 & -4.4 \\
\hline $1 / \exp \left(-r_{k}^{\mathrm{H}} / 7 \AA\right)$ & -4.2 & -12.0 & -20.7 & -30.8 & -40.0 & -50.7 & -55.1 & -50.9 & -41.4 & -29.9 & -18.8 & -9.0 & -3.4 \\
\hline $1 / \exp \left(-r_{k}^{\mathrm{H}} / 10 \AA\right)$ & -4.6 & -10.8 & -18.1 & -26.3 & -33.9 & -42.7 & -46.2 & -41.3 & -32.5 & -22.7 & -13.8 & -6.3 & -1.9 \\
\hline $\operatorname{sigm}\left(3 \AA-r_{k}^{\mathrm{H}}\right)$ & -3.5 & 0.2 & 6.0 & 13.1 & 20.5 & 31.6 & 39.0 & 37.7 & 34.2 & 24.7 & 17.4 & 10.3 & 7.4 \\
\hline $\operatorname{sigm}\left(4 \AA-r_{k}^{\mathrm{H}}\right)$ & -2.9 & 1.3 & 7.9 & 15.5 & 23.3 & 34.8 & 41.8 & 39.7 & 35.1 & 25.6 & 17.5 & 9.6 & 6.3 \\
\hline $\operatorname{sigm}\left(5 \AA-r_{k}^{\mathrm{H}}\right)$ & -2.0 & 2.8 & 10.1 & 18.2 & 26.2 & 37.9 & 44.2 & 41.0 & 35.4 & 25.9 & 17.2 & 8.4 & 4.9 \\
\hline $\operatorname{sigm}\left(7 \AA-r_{k}^{\mathrm{H}}\right)$ & -0.2 & 5.8 & 13.4 & 22.2 & 30.2 & 41.0 & 46.4 & 41.5 & 34.1 & 25.2 & 15.7 & 6.2 & 2.2 \\
\hline $\operatorname{sigm}\left(10 \AA-r_{k}^{H}\right)$ & 1.7 & 7.6 & 15.3 & 24.1 & 31.6 & 40.7 & 45.2 & 39.7 & 31.9 & 23.7 & 14.5 & 5.5 & 1.1 \\
\hline $1 / \operatorname{sigm}\left(3 \AA-r_{k}^{\mathrm{H}}\right)$ & 1.7 & -2.9 & -8.9 & -17.5 & -25.8 & -37.5 & -42.9 & -43.4 & -37.1 & -27.8 & -17.7 & -11.4 & -8.1 \\
\hline $1 / \operatorname{sigm}\left(4 \AA-r_{k}^{\mathrm{H}}\right)$ & 1.4 & -3.7 & -10.2 & -19.3 & -28.4 & -40.7 & -46.5 & -45.8 & -39.1 & -29.2 & -18.6 & -11.3 & -7.5 \\
\hline $1 / \operatorname{sigm}\left(5 \AA-r_{k}^{\mathrm{H}}\right)$ & 0.9 & -4.8 & -12.0 & -21.7 & -31.5 & -44.6 & -50.7 & -48.5 & -41.1 & -30.5 & -19.5 & -10.9 & -6.6 \\
\hline $1 / \operatorname{sigm}\left(7 \AA-r_{k}^{\mathrm{H}}\right)$ & -0.5 & -7.4 & -15.6 & -26.3 & -36.8 & -50.7 & -56.9 & -52.3 & -43.4 & -31.9 & -20.5 & -9.8 & -4.3 \\
\hline $1 / \operatorname{sigm}\left(10 \AA-r_{k}^{\mathrm{H}}\right)$ & -2.6 & -10.1 & -18.8 & -29.9 & -40.1 & -53.2 & -59.2 & -54.3 & -45.0 & -33.1 & -21.4 & -9.6 & -3.0 \\
\hline $\operatorname{hard}\left(3 \AA-r_{k}^{\mathrm{H}}\right)$ & -1.7 & -1.7 & -2.2 & 2.9 & 4.7 & 11.5 & 16.4 & 18.7 & 18.6 & 13.1 & 9.9 & 8.5 & 9.6 \\
\hline $\operatorname{hard}\left(4 \AA-r_{k}^{\mathrm{H}}\right)$ & -5.0 & -2.1 & 1.6 & 7.0 & 12.4 & 21.0 & 27.9 & 28.4 & 27.8 & 20.1 & 15.0 & 9.9 & 8.0 \\
\hline $\operatorname{hard}\left(5 \AA-r_{k}^{\mathrm{H}}\right)$ & -2.6 & -0.7 & 5.3 & 9.8 & 16.1 & 24.8 & 31.2 & 31.6 & 28.4 & 20.3 & 14.5 & 9.3 & 7.4 \\
\hline $\operatorname{hard}\left(7 \AA-r_{k}^{\mathrm{H}}\right)$ & -2.0 & 4.1 & 11.9 & 20.3 & 28.4 & 39.8 & 44.5 & 40.0 & 33.0 & 23.8 & 14.7 & 5.7 & 2.3 \\
\hline $\operatorname{hard}\left(10 \AA-r_{k}^{\mathrm{H}}\right)$ & 1.7 & 7.6 & 14.7 & 23.1 & 30.6 & 39.4 & 44.3 & 38.5 & 31.1 & 23.3 & 14.1 & 5.2 & 1.0 \\
\hline $\exp \left(-r_{k}^{\mathrm{N}} / 3 \AA\right)(\mathrm{BB})$ & 2.1 & 7.6 & 16.2 & 25.9 & 34.9 & 43.6 & 48.4 & 43.4 & 35.5 & 25.6 & 15.7 & 6.2 & 0.7 \\
\hline $1 / \operatorname{hard}\left(7 \AA-r_{k}^{C}\right)($ all $)$ & -2.1 & -8.9 & -18.4 & -29.5 & -40.5 & -52.4 & -52.8 & -41.3 & -29.6 & -17.7 & -7.3 & 0.7 & 4.3 \\
\hline$D_{\text {com }}$ & -3.4 & -10.3 & -19.3 & -27.9 & -33.7 & -38.7 & -38.8 & -32.6 & -25.3 & -17.4 & -8.8 & -1.6 & 2.1 \\
\hline Tail, $M=1$ & -7.4 & -8.7 & -10.6 & -14.1 & -20.5 & -21.7 & -36.5 & -16.3 & -9.1 & -2.8 & 2.3 & 2.8 & 3.9 \\
\hline Tail, $M=2$ & -7.4 & -9.6 & -11.9 & -15.1 & -21.2 & -40.1 & -42.3 & -22.0 & -8.3 & -0.5 & 3.6 & 4.8 & 5.5 \\
\hline Tail, $M=3$ & -6.5 & -9.5 & -12.4 & -15.8 & -36.6 & -41.9 & -41.8 & -26.7 & -10.9 & 1.3 & 5.2 & 6.2 & 8.0 \\
\hline Tail, $M=4$ & -4.7 & -8.5 & -12.2 & -31.2 & -38.5 & -41.1 & -39.3 & -26.4 & -15.9 & -2.2 & 6.5 & 8.4 & 9.2 \\
\hline Tail, $M=5$ & -2.9 & -6.7 & -26.3 & -33.5 & -38.1 & -38.7 & -36.5 & -25.1 & -17.0 & -8.2 & 3.5 & 9.5 & 10.5 \\
\hline Tail, $M=6$ & -1.9 & -20.0 & -27.5 & -32.7 & -35.8 & -35.9 & -33.7 & -23.6 & -17.0 & -9.6 & -2.7 & 6.1 & 10.7 \\
\hline DSSPcont $\mathrm{G}$ & -7.2 & -9.3 & -13.4 & -10.2 & -4.9 & -1.9 & -0.1 & -2.2 & -4.7 & -2.6 & 0.3 & 3.1 & 1.2 \\
\hline DSSPcont H & -5.4 & 0.1 & 5.7 & 12.1 & 16.2 & 19.1 & 22.7 & 21.2 & 16.9 & 11.8 & 6.5 & 2.6 & -1.1 \\
\hline DSSPcont I & -6.4 & -14.3 & -14.2 & -3.4 & -0.5 & 1.0 & 1.2 & 0.9 & -0.5 & -1.7 & 2.2 & 3.0 & 1.4 \\
\hline DSSPcont $\mathrm{T}$ & 3.3 & 1.1 & -1.5 & -6.0 & -3.8 & 0.5 & 1.4 & 0.1 & 2.1 & 2.3 & 3.7 & 7.7 & 8.9 \\
\hline DSSPcont E & 2.7 & 5.5 & 7.7 & 10.7 & 14.2 & 16.7 & 14.9 & 9.6 & 5.6 & 0.6 & -2.4 & -0.8 & -1.4 \\
\hline
\end{tabular}


Table 2 (continued)

\begin{tabular}{|c|c|c|c|c|c|c|c|c|c|c|c|c|c|}
\hline \multirow[t]{2}{*}{ Feature description } & \multicolumn{13}{|c|}{ Position, $k$} \\
\hline & -6 & -5 & -4 & -3 & -2 & -1 & 0 & +1 & +2 & +3 & +4 & +5 & +6 \\
\hline DSSPcont B & 4.2 & 2.8 & 2.4 & 1.8 & -0.1 & 0.9 & 3.4 & -2.9 & -0.1 & -4.1 & -5.7 & -4.1 & -7.5 \\
\hline DSSPcont S & 8.0 & 2.8 & 0.8 & -8.5 & -14.8 & -15.6 & -20.1 & -14.6 & -8.4 & -4.4 & 0.9 & 0.9 & 2.6 \\
\hline DSSPcont L & -2.1 & -4.3 & -8.8 & -12.7 & -21.4 & -33.2 & $-\mathbf{3 3 . 8}$ & -26.9 & -22.4 & -12.4 & -8.6 & -10.0 & -4.9 \\
\hline Secondary & -3.2 & 1.8 & 7.0 & 16.1 & 27.3 & 37.7 & $\underline{41.5}$ & 32.0 & 24.3 & 13.3 & 6.6 & 7.8 & 2.4 \\
\hline Bend $(-1,0,1)$ & 1.6 & 0.9 & -0.9 & -4.2 & -8.6 & -15.7 & -16.7 & -14.6 & -13.2 & -11.1 & -9.8 & -9.0 & -5.1 \\
\hline Bend $(-2,0,2)$ & 3.3 & 2.8 & 1.3 & -1.5 & -5.1 & -8.3 & $\overline{-10.1}$ & -12.2 & -12.0 & -11.4 & -10.8 & -10.2 & -6.4 \\
\hline Loop left & -3.8 & -5.8 & -9.5 & -14.5 & -22.6 & -28.6 & -32.0 & -37.6 & -35.5 & -33.9 & -30.3 & -30.1 & -27.5 \\
\hline Loop right & -14.4 & -19.0 & -25.7 & -36.0 & -40.4 & -37.6 & -37.6 & -17.4 & -5.3 & 1.1 & 3.9 & 3.8 & 4.3 \\
\hline Residue size & -6.4 & -1.5 & -0.2 & 4.1 & 6.4 & 9.0 & 10.0 & 7.2 & 6.4 & 2.3 & -1.2 & -4.1 & -6.0 \\
\hline BB H-bonds energy & 5.1 & -2.5 & -7.8 & -15.3 & -22.3 & -27.1 & -34.1 & -31.3 & -26.6 & -16.0 & -10.0 & -2.8 & 0.8 \\
\hline
\end{tabular}

included in the summation. When $\mathrm{X}=\mathrm{O}$, the reference atom is the carbonyl oxygen of the $(i+k)$ th residue and heavy atoms in the $(i+k)$ th and $(i+k+1)$ th residues are not included in the summation. When $\mathrm{X}=\mathrm{C}$, the reference atom is the $\mathrm{C}^{\alpha}$ atom of the residue. The modifier "(BB)" indicates that the summation involves only the atoms that are part of the backbone $\left(\mathrm{N}, \mathrm{C}_{\alpha}, \mathrm{C}^{\prime}\right)$. The modifier "(all)" indicates that the summation extends over all residues. The function $\operatorname{hard}(x)$ is defined by,

$\operatorname{hard}(x)= \begin{cases}1, & \text { if } x \geqslant 0 \\ 0, & \text { if } x<0\end{cases}$

The notation " $1 / \ldots$ " indicates the inverse.

Some of these features are similar to quantities used by other authors. For example, "exp $\left(-r_{0}^{\mathrm{H}} / 1 \AA\right)$ " and "exp $\left(-r_{-1}^{\mathrm{O}} / 1 \AA\right)$ " are the components of formula used by Zhang-Brüschweiler to predict $S^{2}$ [26]. In addition, " $1 / \operatorname{hard}\left(7 \AA-r_{0}^{\mathrm{C}}\right)$ (all)" corresponds to the function used by Halle to interpret crystallographic B-factors [6].

\subsection{Optimization}

To find the optimal, in the least-squared sense, values of the parameters $\mathrm{W}_{1}, \mathrm{~W}_{2}, \vec{b}_{1}$, and $\vec{b}_{2}$, the Levenberg-Marquardt algorithm was used $[32,33]$. The learning process was cross-validated by iteratively selecting one of the proteins from the data set, also excluding its relatives from the data set, allowing the network to learn $S^{2}$ from the remaining set and calculating the Pearson correlation between experimental and predicted values of $S^{2}$. This step was repeated for all proteins in the dataset. The average correlation was used as a measure of the quality of the prediction process.

\subsection{Independent data}

Experimental generalized order parameters have been reported for B3 domain of staphyloccocal protein $G$ (GB3) [34], the villin headpiece domain (HP67) [35], the holo frenolicin acyl carrier protein (fren ACP) [36], and Escherichia coli ribonuclease H (RNaseH) [37-40]. Generalized order parameters were predicted using the structural coordinates from PDB files 1IGD, 1QQV, 1OR5, and $2 \mathrm{RN} 2$, respectively. Experimental generalized order parameters have been reported for wild-type and loopswap mutants of the 10th fibronectin type III domain of the protein fibronectin (fnfn10) and of the third fibronectin type III domain of the protein tenascin $(\operatorname{tnfn} 3)$ [41]. The mutants were constructed by interchange of the $\mathrm{CC}^{\prime}$ and FG loops between fnfn 10 and $\operatorname{tnfn} 3$. Generalized order parameters were predicted using structural models described elsewhere [41]. GB3, HP67, fren ACP, RNaseH, fnfn10, and tnfn3 are not similar to any of the proteins used to develop the prediction method.

\section{Results}

\subsection{Statistical analysis and validation}

Correlations between some of the features we examined and the normalized squared order parameters for all proteins in the sample set are summarized in Table 2. The average correlations determined by analyzing each protein independently are shown in Table 3.

Because of the finite size of the training set, supervised learning can be subject to the problem of overfitting/overtraining. Therefore, the number of adjustable parameters and, consequently, the number of features used by the network, must be limited. After some experimentation, we decided to use just six features and no hidden layer, i.e. a simple perceptron that can only capture linear correlations (due to technical issues, this perceptron was realized by using a single hidden unit in a two-layer feed-forward network). The cells of Tables 2 and 3 corresponding to the features presently incorporated into the model are marked in bold font and underlined. Features utilized in the final model may not necessarily correspond to features with the highest correlation in Tables 2 and 3. The network was trained using least squares minimization of the difference between the experimental and predicted values of $S^{2}$; the reported correlation coefficients are descriptive statistics. The various features are mutually cross-correlated to different extents and such correlations are not exhibited in the tables.

The optimized values of the model parameters are shown in Table 4 . The results of cross-validating the opti- 
Table 3

Average correlations $100 C_{x y}$ between features and $S^{2}$

\begin{tabular}{|c|c|c|c|c|c|c|c|c|c|c|c|c|c|}
\hline \multirow[t]{2}{*}{ Feature description } & \multicolumn{13}{|c|}{ Position, $k$} \\
\hline & -6 & -5 & -4 & -3 & -2 & -1 & 0 & +1 & +2 & +3 & +4 & +5 & +6 \\
\hline$\overline{\exp \left(-r_{k}^{\mathrm{O}} / 1 \AA\right)}$ & 5.0 & 13.6 & 23.0 & 29.1 & 34.4 & 38.8 & 32.8 & 21.5 & 12.2 & 1.8 & -3.7 & -11.8 & -11.7 \\
\hline $\exp \left(-r_{k}^{\mathrm{O}} / 2 \AA\right)$ & 8.1 & 16.9 & 27.2 & 33.7 & 40.5 & 44.8 & 38.8 & 24.1 & 13.6 & 1.7 & -6.5 & -14.6 & -14.0 \\
\hline $\exp \left(-r_{k}^{\mathrm{O}} / 3 \AA\right)$ & 9.2 & 18.0 & 28.1 & 34.7 & 41.6 & $\underline{45.8}$ & 40.2 & 24.9 & 14.2 & 2.2 & -7.3 & -15.2 & -14.6 \\
\hline $\exp \left(-r_{k}^{\mathrm{O}} / 4 \AA\right)$ & 9.5 & 18.3 & 28.2 & 34.9 & 41.8 & $\overline{45.9}$ & 40.7 & 25.4 & 14.6 & 2.7 & -7.4 & -15.1 & -14.7 \\
\hline $\exp \left(-r_{k}^{\mathrm{O}} / 5 \AA\right)$ & 9.5 & 18.3 & 28.1 & 34.9 & 41.8 & 45.9 & 40.9 & 25.7 & 14.9 & 3.1 & -7.2 & -14.9 & -14.6 \\
\hline $\exp \left(-r_{k}^{\mathrm{O}} / 7 \AA\right)$ & 9.4 & 18.2 & 28.0 & 34.9 & 41.7 & 45.8 & 41.1 & 26.1 & 15.4 & 3.7 & -7.0 & -14.4 & -14.4 \\
\hline $\exp \left(-r_{k}^{\mathrm{O}} / 10 \AA\right)$ & 9.3 & 18.0 & 27.8 & 34.8 & 41.7 & 45.8 & 41.3 & 26.5 & 15.8 & 4.2 & -6.7 & -13.9 & -14.2 \\
\hline $1 / \exp \left(-r_{k}^{\mathrm{O}} / 1 \AA\right)$ & 5.0 & 13.6 & 23.0 & 29.1 & 34.4 & 38.8 & 32.8 & 21.5 & 12.2 & 1.8 & -3.7 & -11.8 & -11.7 \\
\hline $1 / \exp \left(-r_{k}^{\mathrm{O}} / 2 \AA\right)$ & -6.4 & -14.0 & -23.0 & -33.1 & -42.3 & -51.8 & -45.2 & -26.1 & -16.9 & -5.0 & 4.8 & 12.2 & 11.2 \\
\hline $1 / \exp \left(-r_{k}^{\mathrm{O}} / 3 \AA\right)$ & -8.4 & -16.1 & -25.9 & -36.1 & -45.1 & -53.9 & -48.1 & -29.1 & -19.2 & -6.0 & 5.0 & 12.4 & 12.4 \\
\hline $1 / \exp \left(-r_{k}^{\mathrm{O}} / 4 \AA\right)$ & -9.2 & -17.0 & -27.2 & -37.2 & -45.9 & -53.9 & -48.7 & -30.3 & -20.2 & -6.7 & 5.0 & 12.4 & 12.9 \\
\hline $1 / \exp \left(-r_{k}^{\mathrm{O}} / 5 \AA\right)$ & -9.5 & -17.4 & -27.8 & -37.6 & -46.1 & -53.5 & -48.6 & -30.7 & -20.5 & -7.0 & 5.0 & 12.4 & 13.2 \\
\hline $1 / \exp \left(-r_{k}^{\mathrm{O}} / 7 \AA\right)$ & -9.7 & -17.7 & -28.3 & -37.7 & -45.7 & -52.4 & -47.8 & -30.7 & -20.5 & -7.1 & 5.0 & 12.3 & 13.3 \\
\hline $1 / \exp \left(-r_{k}^{\mathrm{O}} / 10 \AA\right)$ & -9.6 & -17.8 & -28.4 & -37.4 & -45.1 & -51.1 & -46.6 & -30.2 & -19.9 & -7.0 & 5.1 & 12.3 & 13.4 \\
\hline $\exp \left(-r_{k}^{\mathrm{H}} / 1 \AA\right)$ & -4.3 & -1.2 & 2.7 & 8.1 & 15.7 & 26.6 & 36.0 & 35.0 & 33.9 & 23.0 & 18.3 & 9.1 & 8.7 \\
\hline $\exp \left(-r_{k}^{\mathrm{H}} / 2 \AA\right)$ & -3.0 & 2.5 & 9.9 & 17.8 & 26.2 & 39.2 & $\underline{48.3}$ & 43.0 & 36.9 & 24.5 & 15.4 & 4.0 & 1.1 \\
\hline $\exp \left(-r_{k}^{\mathrm{H}} / 3 \AA\right)$ & -2.0 & 4.2 & 12.4 & 21.0 & 29.4 & 41.5 & $\overline{49.4}$ & 42.8 & 35.1 & 23.0 & 12.9 & 1.6 & -2.2 \\
\hline $\exp \left(-r_{k}^{\mathrm{H}} / 4 \AA\right)$ & -1.6 & 4.7 & 13.2 & 21.9 & 30.2 & 41.7 & 49.0 & 42.1 & 33.9 & 22.3 & 11.8 & 0.9 & -3.4 \\
\hline $\exp \left(-r_{k}^{\mathrm{H}} / 5 \AA\right)$ & -1.4 & 4.9 & 13.4 & 22.2 & 30.5 & 41.6 & 48.6 & 41.6 & 33.3 & 21.9 & 11.3 & 0.7 & -3.8 \\
\hline $\exp \left(-r_{k}^{\mathrm{H}} / 7 \AA\right)$ & -1.4 & 4.9 & 13.5 & 22.4 & 30.6 & 41.4 & 48.1 & 41.2 & 32.8 & 21.6 & 10.9 & 0.7 & -4.1 \\
\hline $\exp \left(-r_{k}^{\hat{H}} / 10 \AA ̊\right)$ & -1.4 & 4.9 & 13.4 & 22.4 & 30.6 & 41.3 & 47.7 & 40.9 & 32.5 & 21.5 & 10.7 & 0.8 & -4.2 \\
\hline $1 / \exp \left(-r_{k}^{\mathrm{H}} / 1 \AA\right)$ & 2.9 & 0.2 & -4.2 & -9.8 & -17.8 & -31.4 & -39.3 & -39.0 & -37.1 & -23.8 & -16.9 & -8.2 & -5.9 \\
\hline $1 / \exp \left(-r_{k}^{\mathrm{H}} / 2 \AA\right)$ & 2.5 & -2.5 & -9.3 & -17.6 & -27.4 & -43.0 & -52.5 & -47.9 & -42.8 & -27.2 & -16.2 & -5.4 & -1.2 \\
\hline $1 / \exp \left(-r_{k}^{\mathrm{H}} / 3 \AA\right)$ & 1.8 & -3.9 & -11.7 & -21.6 & -31.3 & -46.5 & -55.7 & -49.5 & -42.5 & -27.0 & -15.0 & -3.7 & 1.5 \\
\hline $1 / \exp \left(-r_{k}^{\mathrm{H}} / 4 \AA\right)$ & 1.5 & -4.5 & -12.7 & -23.0 & -32.6 & -47.1 & -55.8 & -49.1 & -41.4 & -26.7 & -14.3 & -3.1 & 2.6 \\
\hline $1 / \exp \left(-r_{k}^{\mathrm{H}} / 5 \AA\right)$ & 1.3 & -4.8 & -13.1 & -23.6 & -33.1 & -47.0 & -55.2 & -48.3 & -40.4 & -26.4 & -13.9 & -2.8 & 3.1 \\
\hline $1 / \exp \left(-r_{k}^{\mathrm{H}} / 7 \AA\right)$ & 1.2 & -5.0 & -13.5 & -24.0 & -33.2 & -46.2 & -53.8 & -46.9 & -38.9 & -25.8 & -13.4 & -2.6 & 3.4 \\
\hline $1 / \exp \left(-r_{k}^{\mathrm{H}} / 10 \AA ̊\right)$ & 1.3 & -5.0 & -13.6 & -23.9 & -32.9 & -45.2 & -52.3 & -45.5 & -37.3 & -24.9 & -12.7 & -2.3 & 3.6 \\
\hline $\operatorname{sigm}\left(3 \AA-r_{k}^{\mathrm{H}}\right)$ & -4.2 & -0.5 & 5.1 & 11.1 & 19.2 & 31.9 & 41.5 & 38.9 & 36.2 & 24.5 & 18.2 & 7.9 & 6.4 \\
\hline $\operatorname{sigm}\left(4 \AA-r_{k}^{\mathrm{H}}\right)$ & -4.1 & 0.3 & 6.9 & 13.4 & 21.6 & 34.8 & 44.2 & 40.4 & 36.4 & 24.5 & 17.2 & 6.2 & 4.2 \\
\hline $\operatorname{sigm}\left(5 \AA-r_{k}^{\mathrm{H}}\right)$ & -3.6 & 1.7 & 9.0 & 16.1 & 24.3 & 37.6 & 46.4 & 41.2 & 35.8 & 23.8 & 15.4 & 4.1 & 1.5 \\
\hline $\operatorname{sigm}\left(7 \AA-r^{H}\right)$ & -2.2 & 4.4 & 12.3 & 20.5 & 28.2 & 40.5 & 48.1 & 41.2 & 33.3 & 21.8 & 11.9 & 0.6 & -2.8 \\
\hline $\operatorname{sigm}\left(10 \AA-r_{k}^{\mathrm{H}}\right)$ & -0.1 & 5.6 & 13.8 & 22.4 & 29.7 & 40.2 & 46.9 & 39.3 & 30.8 & 20.0 & 9.6 & -1.0 & -5.3 \\
\hline $1 / \operatorname{sigm}\left(3 \AA-r_{k}^{\mathrm{H}}\right)$ & 3.0 & -0.4 & -5.5 & -11.8 & -20.6 & -35.1 & -44.0 & -42.0 & -39.5 & -25.6 & -17.1 & -7.5 & -4.7 \\
\hline $1 / \operatorname{sigm}\left(4 \AA-r_{k}^{\mathrm{H}}\right)$ & 3.1 & -1.0 & -6.8 & -13.7 & -22.8 & -37.9 & -47.2 & -43.8 & -40.6 & -26.3 & -16.9 & -6.6 & -3.4 \\
\hline $1 / \operatorname{sigm}\left(5 \AA-r_{k}^{\mathrm{H}}\right)$ & 3.1 & -1.8 & -8.4 & -16.2 & -25.6 & -41.2 & -50.7 & -45.8 & -41.2 & -26.4 & -16.1 & -5.3 & -1.7 \\
\hline $1 / \operatorname{sigm}\left(7 \AA-r_{k}^{\mathrm{H}}\right)$ & 2.3 & -3.8 & -11.3 & -21.0 & -30.1 & -45.7 & -55.3 & -48.1 & -40.8 & -25.6 & -13.9 & -2.8 & 1.9 \\
\hline $1 / \operatorname{sigm}\left(10 \AA-r_{k}^{\mathrm{H}}\right)$ & 0.5 & -5.2 & -13.2 & -23.9 & -32.4 & -46.5 & -55.6 & -47.9 & -39.4 & -24.9 & -12.7 & -1.6 & 4.3 \\
\hline $\operatorname{hard}\left(3 \AA-r_{k}^{\mathrm{H}}\right)$ & -0.2 & -0.8 & -3.3 & 0.7 & 4.4 & 12.6 & 17.6 & 20.8 & 21.5 & 14.1 & 12.3 & 9.3 & 12.9 \\
\hline $\operatorname{hard}\left(4 \AA-r_{k}^{\mathrm{H}}\right)$ & -5.2 & -2.8 & 0.6 & 5.6 & 13.2 & 22.6 & 31.5 & 31.5 & 32.3 & 22.7 & 19.1 & 10.5 & 9.8 \\
\hline $\operatorname{hard}\left(5 \AA-r_{k}^{\mathrm{H}}\right)$ & -3.7 & -1.5 & 4.6 & 7.8 & 15.3 & 25.5 & 33.7 & 32.7 & 31.4 & 21.3 & 16.4 & 7.3 & 6.7 \\
\hline $\operatorname{hard}\left(7 \AA-r_{k}^{\mathrm{H}}\right)$ & -4.6 & 3.0 & 11.2 & 19.1 & 26.8 & 39.3 & 46.3 & 39.9 & 32.7 & 20.7 & 11.6 & 0.8 & -2.0 \\
\hline $\operatorname{hard}\left(10 \AA-r_{k}^{\mathrm{H}}\right)$ & 0.1 & 5.8 & 13.3 & 21.4 & 28.5 & 38.8 & 45.8 & 38.2 & 30.1 & 19.8 & 9.5 & -1.2 & -5.0 \\
\hline $\exp \left(-r_{k}^{\mathrm{N}} / 3 \AA\right)(\mathrm{BB})$ & -1.9 & 4.8 & 13.8 & 23.5 & 33.3 & 44.7 & 50.6 & 43.4 & 34.1 & 22.1 & 11.3 & 0.5 & -5.2 \\
\hline $1 / \operatorname{hard}\left(7 \AA-r_{k}^{\mathrm{C}}\right)($ all $)$ & -0.1 & -7.6 & -16.5 & -26.8 & -38.1 & -53.5 & -51.1 & -36.7 & -25.6 & -12.8 & -1.5 & 7.4 & 10.0 \\
\hline$D_{\text {com }}$ & -3.3 & -10.3 & -19.3 & -28.1 & -34.6 & -42.2 & -42.7 & -32.2 & -22.8 & -12.8 & -1.4 & 7.0 & 10.1 \\
\hline Tail, $M=1$ & -5.7 & -6.0 & -7.6 & -10.6 & -17.4 & -23.4 & -40.8 & -12.3 & -10.8 & -5.3 & 3.1 & 1.7 & 4.6 \\
\hline Tail, $M=2$ & -5.3 & -6.5 & -8.5 & -10.8 & -17.3 & -41.0 & -45.1 & -26.1 & -9.9 & -3.3 & 2.7 & 4.3 & 6.4 \\
\hline Tail, $M=3$ & -4.8 & -6.0 & -8.3 & -11.1 & -33.0 & -41.3 & -42.1 & -29.7 & -15.4 & -1.2 & 5.1 & 6.1 & 9.6 \\
\hline Tail, $M=4$ & -3.0 & -5.6 & -7.7 & -27.2 & -34.1 & -39.7 & -38.5 & -27.4 & -19.4 & -6.0 & 6.7 & 8.9 & 11.2 \\
\hline Tail, $M=5$ & -1.2 & -3.9 & -22.7 & -28.6 & -33.9 & -36.5 & $\overline{-34.8}$ & -25.1 & -18.5 & -10.4 & 2.2 & 10.6 & 12.6 \\
\hline Tail, $M=6$ & -0.9 & -17.3 & -23.4 & -28.4 & -31.3 & -33.3 & -31.7 & -22.5 & -17.1 & -9.7 & -2.6 & 5.3 & 12.8 \\
\hline DSSPcont $\mathrm{G}$ & -5.1 & -9.0 & -15.2 & -11.6 & -5.4 & -2.8 & -0.7 & -2.1 & -2.7 & -0.4 & 0.8 & 6.5 & 4.6 \\
\hline DSSPcont $\mathrm{H}$ & -6.4 & -0.4 & 5.9 & 13.1 & 17.9 & 22.0 & 26.3 & 25.3 & 21.4 & 16.5 & 10.7 & 6.5 & 2.4 \\
\hline DSSPcont I & -3.4 & -6.0 & -5.7 & -2.5 & -0.4 & -2.4 & 0.0 & 0.3 & 0.9 & 0.8 & 2.0 & 3.5 & 2.9 \\
\hline DSSPcont $\mathrm{T}$ & 2.1 & -1.4 & -3.1 & -9.7 & -7.5 & -1.5 & -1.1 & -3.7 & -0.3 & 2.3 & 4.5 & 9.3 & 10.2 \\
\hline DSSPcont E & 2.7 & 5.9 & 7.3 & 10.1 & 12.8 & 14.9 & 11.6 & 4.9 & -0.1 & -6.9 & -12.4 & -9.4 & -9.3 \\
\hline
\end{tabular}


Table 3 (continued)

\begin{tabular}{|c|c|c|c|c|c|c|c|c|c|c|c|c|c|}
\hline \multirow[t]{2}{*}{ Feature description } & \multicolumn{13}{|c|}{ Position, $k$} \\
\hline & -6 & -5 & -4 & -3 & -2 & -1 & 0 & +1 & +2 & +3 & +4 & +5 & +6 \\
\hline DSSPcont B & 5.5 & 3.9 & 3.1 & 1.4 & -0.3 & -0.0 & 3.3 & 0.4 & 0.6 & -2.8 & -1.5 & 1.9 & -5.5 \\
\hline DSSPcont S & 8.7 & 3.3 & 1.0 & -7.5 & -14.3 & -15.1 & -19.5 & -14.3 & -9.0 & -3.4 & 1.1 & 0.2 & 4.2 \\
\hline DSSPcont L & -0.3 & -1.3 & -6.5 & -11.4 & -21.5 & -36.4 & -37.0 & -27.4 & -19.9 & -10.7 & -1.5 & -4.0 & 1.1 \\
\hline Secondary & -5.4 & -0.8 & 5.0 & 14.5 & 26.5 & 39.2 & $\underline{42.9}$ & 31.4 & 22.0 & 10.5 & 0.7 & 3.5 & -3.0 \\
\hline Bend $(-1,0,1)$ & 2.4 & 1.6 & -0.6 & -4.5 & -10.0 & -21.4 & -23.2 & -20.3 & -18.0 & -16.2 & -13.0 & -11.0 & -7.0 \\
\hline Bend $(-2,0,2)$ & 2.6 & 2.1 & 0.5 & -2.6 & -7.0 & -11.8 & -15.5 & -18.6 & -17.7 & -17.1 & -14.7 & -12.7 & -9.0 \\
\hline Loop left & -0.9 & -1.5 & -4.5 & -8.0 & -14.8 & -22.4 & -24.6 & -24.8 & -16.0 & -12.1 & -9.0 & -7.2 & -6.7 \\
\hline Loop right & -8.6 & -8.3 & -15.4 & -20.9 & -24.3 & -26.4 & -34.8 & -14.4 & -5.7 & -0.8 & 4.7 & 3.7 & 5.2 \\
\hline Residue size & -5.2 & -0.6 & 0.4 & 4.7 & 7.6 & 10.4 & 9.4 & 8.5 & 8.1 & 1.8 & 1.1 & -6.2 & -8.0 \\
\hline BB H-bonds energy & 7.4 & -1.4 & -7.3 & -15.3 & -23.1 & -28.5 & -35.5 & -32.8 & -26.7 & -14.6 & -6.7 & 0.9 & 3.8 \\
\hline
\end{tabular}

Table 4

Optimized weights and bias parameters

\begin{tabular}{llc}
\hline Feature & Parameter & Value \\
\hline $\exp \left(-r_{-1}^{\mathrm{O}} / 3 \AA\right)$ & $\mathrm{W}_{1}(1)$ & -2.56 \\
$\exp \left(-r_{0}^{\mathrm{H}} / 2 \AA\right)$ & $\mathrm{W}_{1}(2)$ & -1.94 \\
Secondary, $k=0$ & $\mathrm{~W}_{1}(3)$ & -0.821 \\
DSSPcont $\mathrm{L}, k=0$ & $\mathrm{~W}_{1}(4)$ & -0.270 \\
Bend $(-1,0,1), k=0$ & $\mathrm{~W}_{1}(5)$ & 0.292 \\
Tail, $M=4, k=0$ & $\mathrm{~W}_{1}(6)$ & 0.730 \\
& $\overrightarrow{b_{1}}$ & 0.652 \\
& $\mathrm{~W}_{2}$ & -4.64 \\
& $\overrightarrow{b_{2}}$ & 2.01 \\
\hline
\end{tabular}

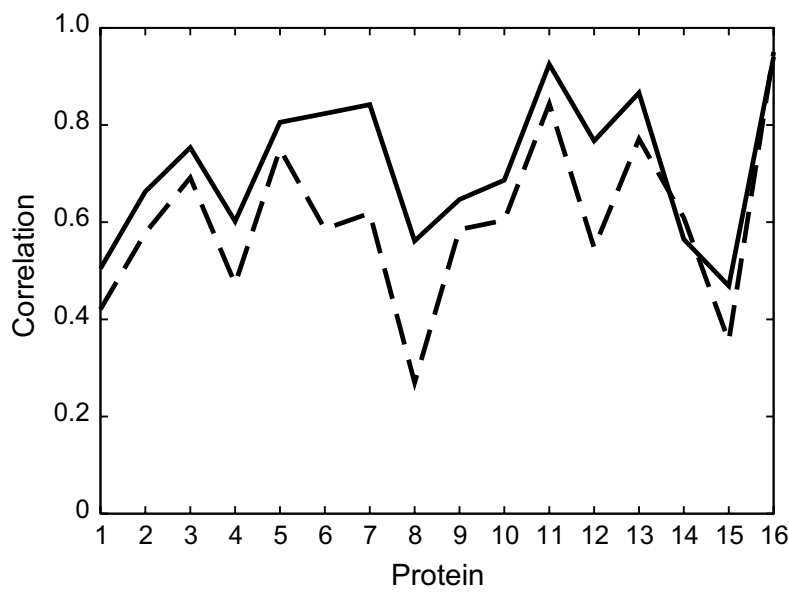

Fig. 1. Correlations between predicted and experimental $S^{2}$. (- - ) ZhangBrüschweiler formula (6); (-) neural network predictions during crossvalidation. Abscissa shows the protein index from Table 1. As noted in Table 1, proteins 2 and 3 and proteins 6 and 7 are closely related.

mized parameters for these features are shown in Fig. 1. Although the predictions themselves are affected by the initial normalization of the $S^{2}$, the average correlation used as the measure of the prediction quality is the same, whether we compare the predictions to the normalized $S^{2}$ or the original unnormalized data. The average correlation between experimental and predicted values of $S^{2}$ during the crossvalidation procedure equals 0.71 with a sample deviation of 0.15 .

\subsection{Sensitivity to structure}

To illustrate the sensitivity of the predictions to details of the protein structure, Fig. 2 shows the $S^{2}$ predictions

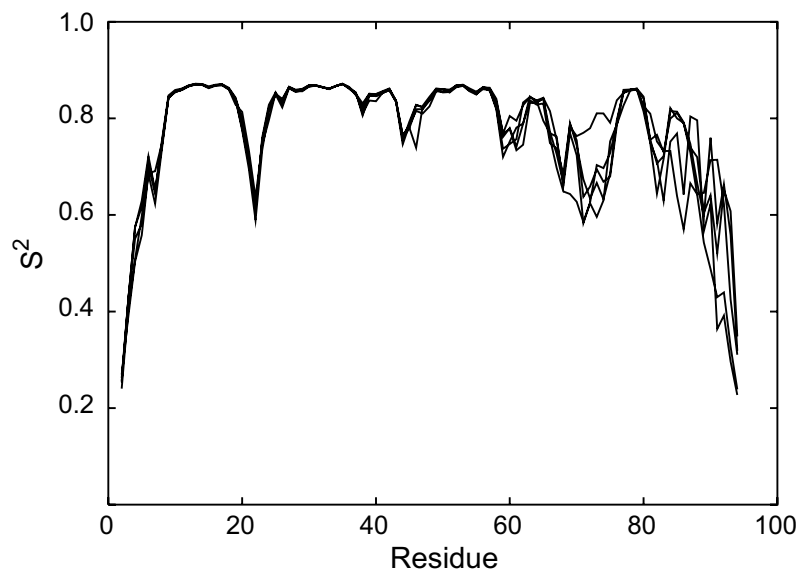

Fig. 2. $S^{2}$ predictions for the first five NMR models of the forkhead domain of the adipocyte-transcription factor freac-11 (S12) (PDB code: 1D5V).

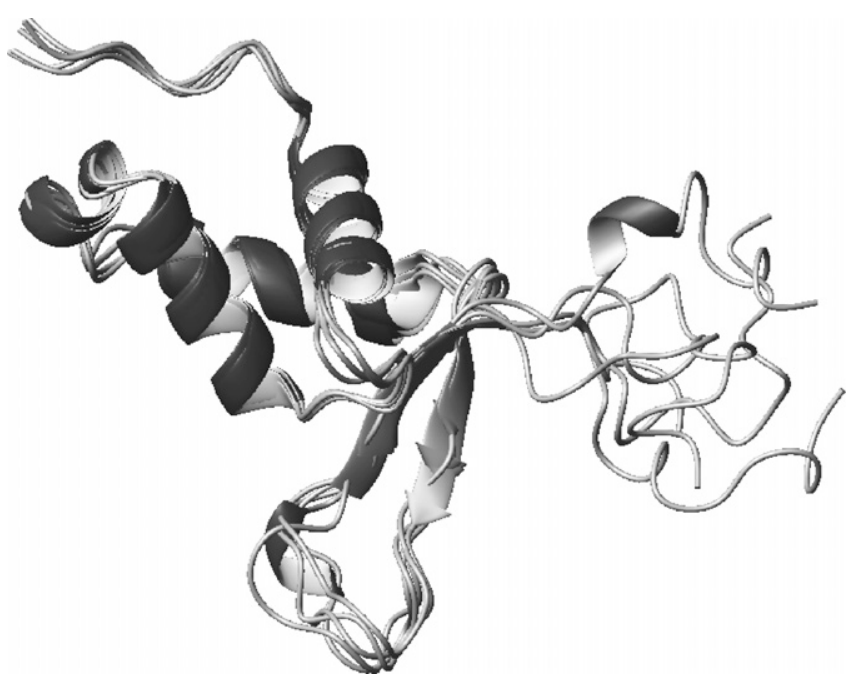

Fig. 3. The backbones of the first five NMR models of the forkhead domain of the adipocyte-transcription factor freac-11 (S12) (PDB code: 1D5V). 

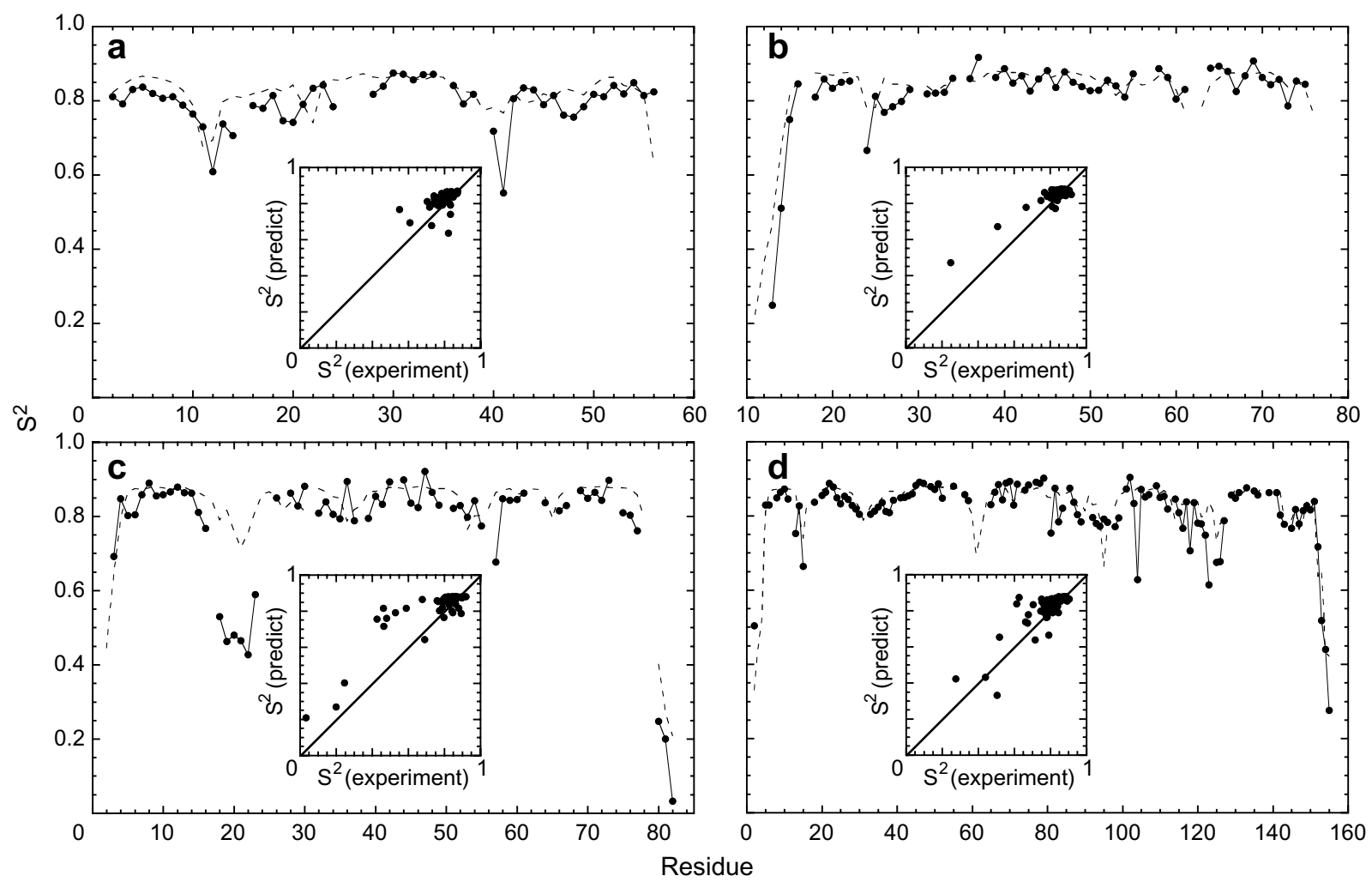

Fig. 4. $S^{2}$ for (a) GB3 (56 residues, $50 S^{2}$ values), (b) HP67 (67 residues, $54 S^{2}$ values), (c) fren ACP (83 residues, $64 S^{2}$ values) and (d) RNase H (155 residues, $120 S^{2}$ values). (-) Experimental measurements and (- - -) neural network predictions are plotted as a function of residue number. (Insets) Predictions are plotted versus experimental values; the $x$ - and $y$-coordinates ranges from 0 to 1 . Predicted values were calculated using structural coordinates from PDB files (a) 1IGD, (b) 1QQV, (c) 1OR5, and (d) 2RN2.

for the first five NMR models of the forkhead domain of the adipocyte-transcription factor freac-11 (also known as S12) [42]. A superposition of the structures is shown in Fig. 3).

\subsection{Prediction for additional proteins}

Backbone dynamics of GB3, HP67, fren ACP, and $\mathrm{RNaseH}$ have been reported in the literature. None of these proteins, nor any homologs, were included in the training set and consequently this set of proteins provides an independent assessment of the performance of the neural network. The experimental and predicted values of $S^{2}$ are shown in Fig. 4. The correlation coefficients between the experimental and predicted data are 0.491, 0.925, 0.867 , and 0.837 for GB3, HP67, fren ACP, and RNaseH, respectively. The root mean-square deviations between experimental and predicted values of $S^{2}$ are $0.061,0.052$, 0.104 , and 0.053 , respectively. The lower correlation for GB3 reflects the absence of residues with markedly low values of $S^{2}$; however, the root mean-square deviation is similar to those for the other proteins. The average correlation coefficient for these four proteins is $0.78 \pm 0.20$ and is similar to the estimate from the cross-validation experiment $(0.71 \pm 0.15)$.

\subsection{Prediction for fibronectin type III domains}

Backbone ${ }^{15} \mathrm{~N} S^{2}$ have been reported for wild-type and two loop swap mutants of fnfn10 and of tnfn3 [41]. The experimental and predicted $S^{2}$ for the wild-type and mutant proteins are shown in Figs. 5 and 6. The wild-type fnfn 10 domain has highly flexible $\mathrm{CC}^{\prime}$ (residues 39-45) and RGD (residues 76-83), shown in Fig. 5a, whereas the wild-type tnfn3 domain has relatively rigid $\mathrm{CC}^{\prime}$ and RGD loops, shown in Fig. 6a. The first fnfn10 mutant, shown in Fig. 5b, substitutes the more rigid $\mathrm{CC}^{\prime}$ loop from tnfn3 for the corresponding loop in fnfn10. The second mutant, shown in Fig. 5c, substitutes the shorter more rigid RGD loop from tnfn3 for the corresponding loop in fnfn10. The predicted values for the two mutant fnfn 10 domains show that the flexibilities of the mutant loops are reduced relative to the native loop sequences. The first tnfn3 mutant, shown in Fig. 6b, substitutes the more flexible $\mathrm{CC}^{\prime}$ loop from fnfn10 for the corresponding loop in tnfn3. The second mutant, shown in Fig. 6c, substitutes the longer more flexible RGD loop from fnfn 10 for the corresponding loop in tnfn3. The predicted values for the two mutant proteins show that the flexibility of the mutant loops is increased relative to the native loop sequences. These results are in qualitative agreement with the experi- 


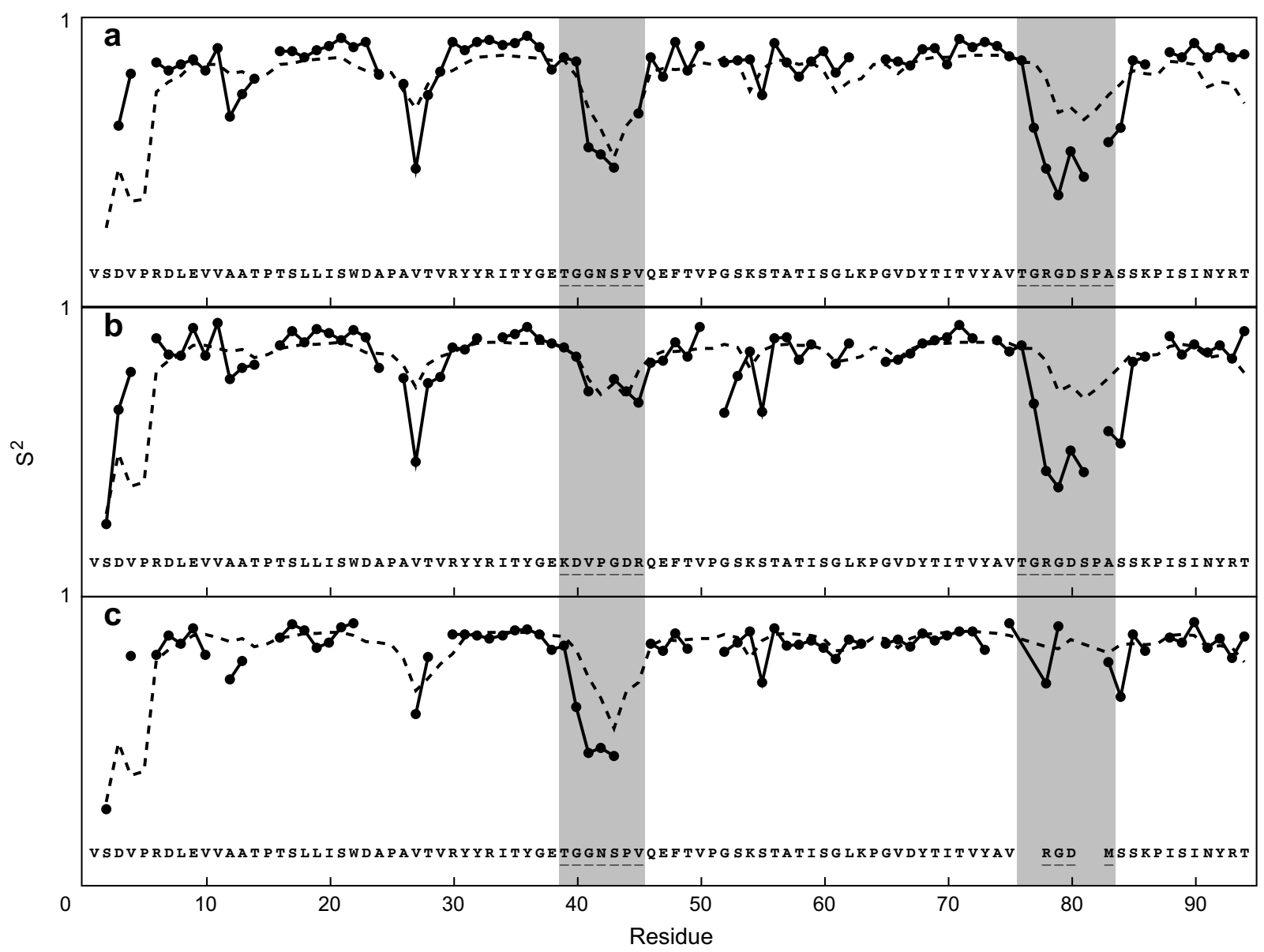

Fig. 5. $S^{2}$ of fnfn10 domain (a) and its mutants (b and c); (-) experimental measurements; (- - -) neural network predictions. The y-coordinate of each figure ranges from 0 to 1 . Residue numbering is based on the wild-type sequence. The location of the $\mathrm{CC}^{\prime}$ and RGD loops is highlighted in gray. The mutations introduced are shown for each variant protein. Structures of the fnfn10 domains used for predictions are described elsewhere [41]. The correlation coefficients between experimental and predicted $S^{2}$ values are (a) 0.620 , (b) 0.625 , and (c) 0.720 .

mental data, although the predictions tend to overestimate the value of $S^{2}$ for the mutant loops for tnfn3.

\section{Discussion and conclusion}

Correlations have been examined for features for residues in positions -6 to +6 relative to the residue for which $S^{2}$ is to be predicted. The correlations shown in Tables 2 and 3 are dominated by residues in the $-1,0$, and +1 positions. Features for residues in positions further away are much less correlated with $S^{2}$.

In the present case, residue size is much less important than local packing density and secondary structure state. The importance of local packing density agrees with the results reported by Zhang and Brüschweiler [26]. Packing density, parameterized differently, was also found to be critical in determining crystallographic B-factors [6]. The relative unimportance of residue size appears to differ from the results of Goodman and coworkers [10]. However, in that earlier study, average values of $S^{2}$ for each amino acid residue were determined first and then correlated with residue side chain volume. This procedure averages over differences in local packing density and secondary structural state and consequently accentuates the dependence on side chain volume compared to the present approach.

The Zhang-Brüschweiler formula for predicting $S^{2}$ is

$S^{2}=\tanh \left[2.656\left(\exp \left(-r_{-1}^{\mathrm{O}} / 1 \AA\right)+0.8 \exp \left(-r_{0}^{\mathrm{H}} / 1 \AA\right)\right]-0.1\right.$.

This formula corresponds to the parametrization expression:

$F=s_{2}\left(\mathrm{~W}_{2} s_{1}\left(\mathrm{~W}_{1} \vec{x}\right)+\vec{b}_{2}\right)$,

and can be thought of as a 2-layer perceptron with two inputs $\left(\exp \left(-r^{O} / 1 \AA\right)\right.$ and $\left.\left.\exp \left(-r^{H} / 1 \AA\right]\right)\right)$. The first layer has a transfer function is $s_{1}(x)=\tanh (x), \mathrm{W}_{1}=[2.656$, $2.125]$, and no bias. The second layer has a transfer function $s_{2}(x)$ equal to the identity operation, $\mathrm{W}_{2}=1$, and a bias $b_{2}=-0.1$. As can be seen from Fig. 1, a consistent improvement in prediction is obtained using the neural network model presented herein compared to the ZhangBrüschweiler formula. Both the present model and the Zhang-Brüschweiler formula use distances to carbonyl oxygen and amide hydrogen atoms as important inputs. The improvement obtained by the neural network results 


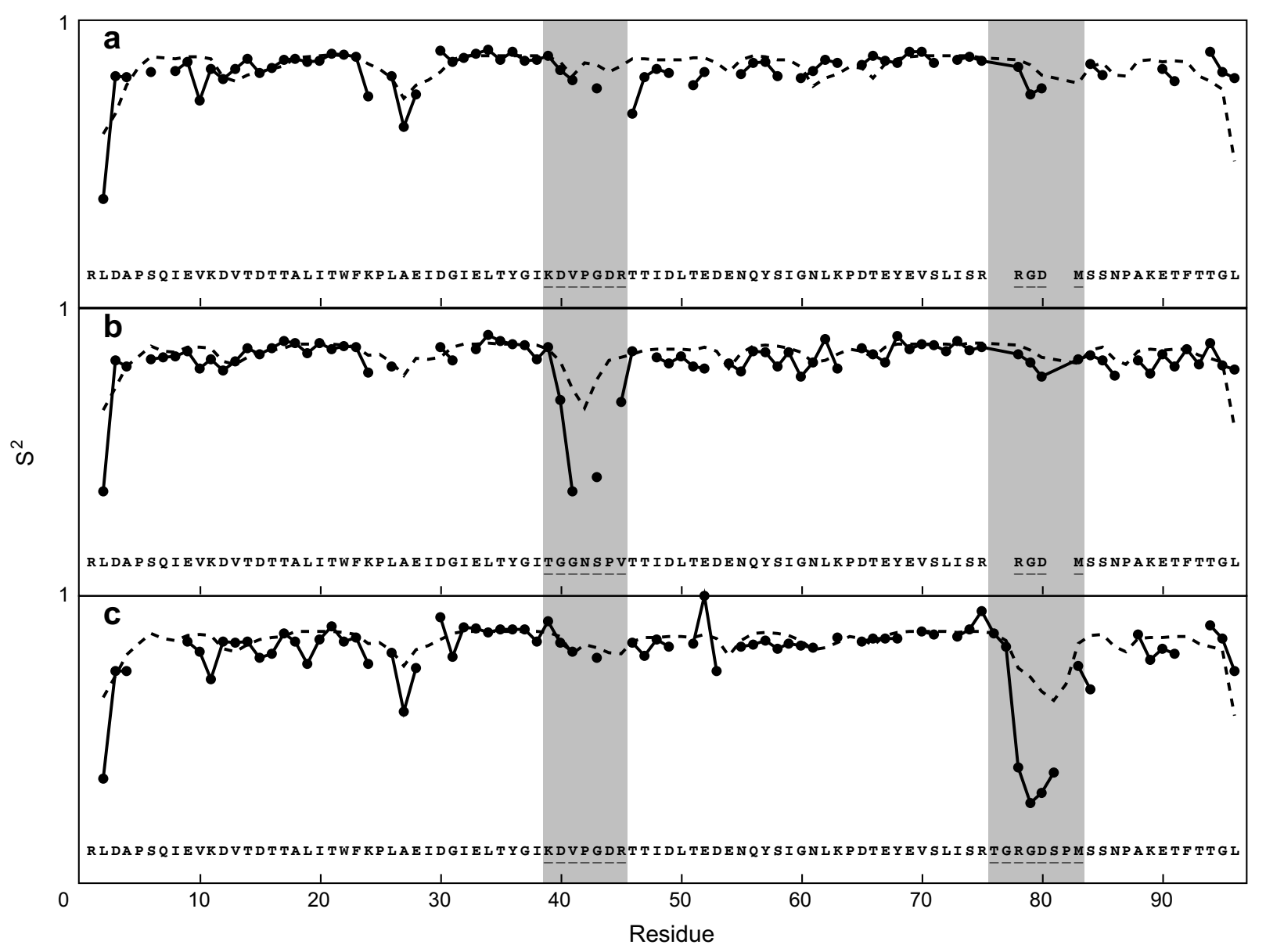

Fig. 6. $S^{2}$ of tnfn3 domain (a) and its mutants (b and c); (-) experimental measurements; (- - -) neural network predictions. The y-coordinate of each section ranges from 0 to 1 . Residue numbering is based on the longest construct, the RGD loop swap variant. The location of the CC' and RGD loops is highlighted in gray. The mutations introduced are shown for each variant protein. Structures of the tnfn 3 domains used for predictions are described elsewhere [41]. The correlation coefficients between experimental and predicted $S^{2}$ values are (a) 0.512 , (b) 0.635 , and (c) 0.759 .

in part from the different characteristic lengths used, 3 and $2 \AA$, for normalizing carbonyl oxygen and amide hydrogen distances, respectively, and in part from additional features in the neural network. Neither the change of the characteristic lengths, nor the addition of any single feature are responsible for most of the accuracy improvement. As shown by Figs. $4-6$, the neural network tends to overestimate the rigidity of loops. This observation suggests that other structural features governing protein conformational dynamics remain to be discovered and parameterized in the future.

Few studies of the accuracy of experimental measurements of $S^{2}$ have been reported. Difficulties in controlling for differences in the models used to fit experimental data is a confounding factor in attempts to determine absolute accuracy of experimental values of $S^{2}$ [21]; consequently, whether further improvements in prediction accuracy are limited by the quality of the experimental $S^{2}$ data or merely by the size of the feature set that can be stably parameterized is unknown.

The predictions obtained for GB3, HP67, fren ACP, $\mathrm{RNaseH}$, and the wild-type and mutant fnfn10 and tnfn3 domains suggest that the parameterization of the neural network is transferable to proteins outside the training set. Furthermore, the predictions obtained for the loopswap mutant fnfn10 and tnfn3 domains suggest that the neural network provides a useful approach for identifying mutant proteins with significantly altered conformational dynamics "in silico" prior to experimental studies.

\section{Acknowledgments}

This work was supported by NIH Grants LM007329 (B.R.) and GM50291 (A.G.P.). Helpful discussions with Ann McDermott (Columbia University) and Wayne Hendrickson (Columbia University) are gratefully acknowledged. The prediction program is available at http:// www.palmer.hs.columbia.edu/software/predictS2.

\section{References}

[1] H. Frauenfelder, S.G. Sligar, P.G. Wolynes, The energy landscapes and motions of proteins, Science 254 (1991) 1598-1603. 
[2] H. Carlson, Protein flexibility and drug design: how to hit a moving target, Curr. Opin. Chem. Biol. 6 (2002) 447-452.

[3] M. Karplus, Molecular dynamics simulations of biomolecules, Acc. Chem. Res. 35 (2002) 321-323.

[4] D. Jacobs, A. Rader, L. Kuhn, M. Thorpe, Protein flexibility predictions using graph theory, Proteins: Struct., Funct., Genet. 44 (2001) 150-165.

[5] P. Doruker, A.R. Atilgan, I. Bahar, namics of proteins predicted by molecular dynamics simulations and analytical approaches: application to $\alpha$-amylase inhibitor, Proteins: Struct., Funct., Genet. 40 (2000) $512-524$.

[6] B. Halle, Flexibility and packing in proteins, Proc. Natl. Acad. Sci. USA 99 (2002) 1274-1279.

[7] A. Schlessinger, B. Rost, Protein flexibility and rigidity predicted from sequence, Proteins 61 (2005) 115-126.

[8] A.G. Palmer, NMR probes of molecular dynamics: overview and comparison with other techniques, Annu. Rev. Biophys. Biomol. Struct. 30 (2001) 129-155.

[9] A.G. Palmer, C.D. Kroenke, J.P. Loria, NMR methods for quantifying microsecond-to-millisecond motions in biological macromolecules, Methods Enzymol. 339 (2001) 204-238.

[10] J.L. Goodman, M.D. Pagel, M.J. Stone, Relationships between protein structure and dynamics from a database of NMR-derived backbone order parameters, J. Mol. Biol. 295 (2000) 963-978.

[11] B.R. Seavey, E.A. Farr, W.M. Westler, J.L. Markley, A relational database for sequence-specific protein NMR data, J. Biomol. NMR 1 (1991) 217-236.

[12] N.A. Farrow, R. Muhandiram, A.U. Singer, S.M. Pascal, C.M. Kay, G. Gish, S.E. Shoelson, T. Pawson, J.D. Forman-Kay, L.E. Kay, Backbone dynamics of a free and a phosphopeptide-complexed Src homology 2 domain studied by ${ }^{15} \mathrm{~N}$ NMR relaxation, Biochemistry 33 (1994) 5984-6003.

[13] L.E. Kay, D.A. Torchia, A. Bax, Backbone dynamics of proteins as studied by nitrogen-15 inverse detected heteronuclear NMR spectroscopy: application to staphylococcal nuclease, Biochemistry 28 (1989) 8972-8979.

[14] D. Muhandiram, T. Yamazaki, B. Sykes, L. Kay, Measurement of ${ }^{2} \mathrm{H} T_{1}$ and $T_{1 \rho}$ relaxation times in uniformly ${ }^{13} \mathrm{C}$-labeled and fractionally ${ }^{2} \mathrm{H}$-labeled proteins in solution, J. Am. Chem. Soc. 117 (1995) 11536-11544.

[15] O. Millet, D.R. Muhandiram, N.R. Skrynnikov, L.E. Kay, Deuterium spin probes of side-chain dynamics in proteins. 1. measurement of five relaxation rates per deuteron in ${ }^{13} \mathrm{C}$-labeled and fractionally ${ }^{2} \mathrm{H}$-enriched proteins in solution, J. Am. Chem. Soc. 124 (2002) 64396448.

[16] G. Lipari, A. Szabo, Model-free approach to the interpretation of nuclear magnetic resonance relaxation in macromolecules, J. Am. Chem. Soc. 104 (1982) 4546-4570.

[17] G. Nodet, D. Abergel, An overview of recent developments in the interpretation and prediction of fast internal protein dynamics, Eur. Biophys. J. 36 (2007) 985-993.

[18] R. Powers, G.M. Clore, D.S. Garrett, A.M. Gronenborn, Relationships between the precision of high-resolution protein NMR structures, solution-order parameters and crystallographic B factors, J. Magn. Reson. B 101 (1993) 325-327.

[19] M.C. Moncrieffe, N. Juranic, M.D. Kemple, J.D. Potter, S. Macura, F.G. Prendergast, Structure-fluorescence correlations in a single tryptophan mutant of carp parvalbumin: solution structure, backbone and side-chain dynamics, J. Mol. Biol. 297 (2000) 147163.

[20] A.G. Palmer, R. Hochstrasser, D.P. Millar, M. Rance, P.E. Wright, Side chain dynamics of a zinc finger peptide characterized by ${ }^{13} \mathrm{C}$ NMR relaxation measurements and fluorescence anisotropy decay, J. Am. Chem. Soc. 115 (1992) 6333-6345.

[21] M. Philippopoulos, A.M. Mandel, A.G. Palmer, C. Lim, Accuracy and precision of NMR relaxation experiments and MD simulations for characterizing protein dynamics, Proteins: Struct., Funct., Genet. 28 (1997) 481-493.

[22] D.C. Chatfield, A. Szabo, B.R. Brooks, Molecular dynamics of staphylococcal nuclease: comparison of simulation with ${ }^{15} \mathrm{Nand}^{13} \mathrm{C}$ NMR relaxation data, J. Am. Chem. Soc. 120 (1998) 5301-5311.

[23] C. Anderson, A. Palmer, S. Brunak, B. Rost, Continuum secondary structure captures protein flexibility, Structure 10 (2002) 175-184.

[24] A. Mittermaier, A.R. Davidson, L.E. Kay, Correlation between ${ }^{2} \mathrm{H}$ NMR side-chain order parameters and sequence conservation in globular proteins, J. Am. Chem. Soc. 125 (2003) 9004-9005.

[25] D. Abergel, G. Bodenhausen, Predicting internal protein dynamics from structures using coupled networks of hindered rotators, J. Chem. Phys. 123 (2005) 204901.

[26] F. Zhang, R. Brüschweiler, Contact model for the prediction of NMR $\mathrm{N}-\mathrm{H}$ order parameters in globular proteins, J. Am. Chem. Soc. 124 (2002) 12654-12655.

[27] D. Ming, R. Brüschweiler, Prediction of methyl-side chain dynamics in proteins, J. Biomol. NMR 29 (2004) 363368.

[28] D. Ming, R. Brüschweiler, Reorientational contact-weighted elastic network model for the prediction of protein dynamics: comparison with NMR relaxation, Biophys. J. 90 (2006) 3382-3388.

[29] H.M. Berman, J. Westbrook, Z. Feng, G. Gilliland, T.N. Bhat, H. Weissig, I.N. Shindyalov, P.E. Bourne, The protein data bank, Nucleic Acids Res. 28 (2000) 235-242.

[30] S. Hykin, Neural Networks: A Comprehensive Foundation, Prentice Hall, Englewood Cliffs, NJ, 1999.

[31] W. Kabsch, C. Sander, How good are predictions of protein secondary structure? FEBS Lett. 155 (1983) 179-182.

[32] K. Levenberg, A method for the solution of certain non-linear problems in least squares, Quart. J. Appl. Math. 2 (1944) 164-168.

[33] M.T. Hagan, M. Menhaj, Training feedforward networks with the Marquardt algorithm, IEEE Trans. Neural Networks 5 (1994) 989993.

[34] J.B. Hall, D. Fushman, Variability of the ${ }^{15} \mathrm{~N}$ chemical shielding tensors in the $\mathrm{B} 3$ domain of protein $\mathrm{G}$ from ${ }^{15} \mathrm{~N}$ relaxation measurements at several fields. implications for backbone order parameters, J. Am. Chem. Soc. 128 (2006) 7855-7870.

[35] M.J. Grey, Y. Tang, E. Alexov, C.J. McKnight, D.P. Raleigh, A.G. Palmer, Characterizing a partially folded intermediate of the villin headpiece domain under non-denaturing conditions: contribution of his41 to the $\mathrm{pH}$-dependent stability of the N-terminal subdomain, J. Mol. Biol. 355 (2006) 1078-1094.

[36] Q. Li, C. Khosla, J.D. Puglisi, C.W. Liu, Solution structure and backbone dynamics of the holo form of the frenolicin acyl carrier protein, Biochemistry 42 (2003) 4648-4657.

[37] K. Yamasaki, M. Saito, M. Oobatake, S. Kanaya, Characterization of the internal motions of Escherichia coli ribonuclease HI by a combination of ${ }^{15} \mathrm{~N}-\mathrm{NMR}$ relaxation analysis and molecular dynamics simulation: examination of dynamic models, Biochemistry 34 (1995) 6587-6601.

[38] A.M. Mandel, M. Akke, A.G. Palmer, Backbone dynamics of Escherichia coli ribonuclease $\mathrm{H}$ : correlations with structure and function of an active enzyme, J. Mol. Biol. 246 (1995) 144-163.

[39] A.M. Mandel, M. Akke, A.G. Palmer, Dynamics of ribonuclease H: temperature dependence of motion on multiple time scales, Biochemistry 35 (1996) 16009-16023.

[40] C.D. Kroenke, M. Rance, A.G. Palmer, Variability of the ${ }^{15} \mathrm{~N}$ chemical shift anisotropy in Escherichia coli ribonuclease $\mathrm{H}$ in solution, J. Am. Chem. Soc. 121 (1999) 10119-10125.

[41] K. Siggers, C. Soto, A.G. Palmer, Conformational dynamics in loop swap mutants of homologous fibronectin type III domains, Biophys. J. 93 (2007) 2447-2456.

[42] M.J.P. van Dongen, A. Cederberg, P. Carlsson, S. Enerbck, M. Wikstrm, Solution structure and dynamics of the DNA-binding domain of the adipocyte-transcription factor FREAC-11, J. Mol. Biol. 296 (2000) 351-359. 